\title{
Publisher's Note: Integrated nanoplasmonic quantum interfaces for room-temperature single-photon sources [Phys. Rev. B 96, 235151 (2017)]
}

Frédéric Peyskens, Darrick Chang, and Dirk Englund

(Q) (Received 5 January 2018; published 11 January 2018)

DOI: 10.1103/PhysRevB.97.039902

This paper was published online on 29 December 2017 with an incorrect order of author names in the author list. The author names and affiliations should read as

Frédéric Peyskens

Quantum Photonics Group, RLE, Massachusetts Institute of Technology, Cambridge, Massachusetts 02139, USA

Darrick Chang

ICFO-Institut de Ciencies Fotoniques, The Barcelona Institute of Science and Technology, 08860 Castelldefels, Barcelona, Spain Dirk Englund

Quantum Photonics Group, RLE, Massachusetts Institute of Technology, Cambridge, Massachusetts 02139, USA

The paper has been corrected as of 5 January 2018. The author names and affiliations is correct in the printed version of the journal. 